\title{
BMJ Open Patient involvement in the implementation of infection prevention and control guidelines and associated interventions: a scoping review
}

\author{
Heloise Fernandes Agreli, ${ }^{1}{ }^{1}$ Michael Murphy, ${ }^{1}$ Sile Creedon, ${ }^{1}$ \\ Cliodhna Ni Bhuachalla, ${ }^{2}$ Deirdre O'Brien, ${ }^{2}$ Dinah Gould, ${ }^{\circ}{ }^{3}$ Eileen Savage, ${ }^{4}$ \\ Fiona Barry, ${ }^{\circ}$ Jonathan Drennan, ${ }^{1}$ Maura P Smiddy, ${ }^{5}$ Sarah Condell, ${ }^{6}$ \\ Sinead Horgan, ${ }^{7}$ Siobhan Murphy, ${ }^{1}$ Teresa Wills, ${ }^{1}$ Aileen Burton, ${ }^{1}$ \\ Josephine Hegarty ${ }^{4}$
}

To cite: Fernandes Agreli $\mathrm{H}$, Murphy M, Creedon S, et al. Patient involvement in the implementation of infection prevention and control guidelines and associated interventions: a scoping review. BMJ Open 2019;9:e025824. doi:10.1136/ bmjopen-2018-025824

- Prepublication history and additional material for this paper are available online. To view these files, please visit the journal online (http://dx.doi org/10.1136/bmjopen-2018025824).

Received 15 August 2018 Revised 7 February 2019 Accepted 12 February 2019

Check for updates

(C) Author(s) (or their employer(s)) 2019. Re-use permitted under CC BY-NC. No commercial re-use. See rights and permissions. Published by BMJ.

For numbered affiliations see end of article.

Correspondence to Dr Heloise Fernandes Agreli; heloiseagreli@gmail.com

\section{ABSTRACT}

Objective To explore patient involvement in the implementation of infection prevention and control (IPC) guidelines and associated interventions.

Design Scoping review.

Methods A methodological framework was followed to identify recent publications on patient involvement in the implementation of IPC guidelines and interventions. Initially, relevant databases were searched to identify pertinent publications (published 2013-2018). Reflecting the scarcity of included studies from these databases, a bidirectional citation chasing approach was used as a second search step. The reference list and citations of all identified papers from databases were searched to generate a full list of relevant references. A grey literature search of Google Scholar was also conducted.

Results From an identified 2078 papers, 14 papers were included in this review. Our findings provide insights into the need for a fundamental change to IPC, from being solely the healthcare professionals (HCPs) responsibility to one that involves a collaborative relationship between HCPs and patients. This change should be underpinned by a clear understanding of patient roles, potential levels of patient involvement in IPC and strategies to overcome barriers to patient involvement focusing on the professional-patient relationship (eg, patient encouragement through multimodal educational strategies and efforts to disperse professional's power).

Conclusions There is limited evidence regarding the best strategies to promote patient involvement in the implementation of IPC interventions and guidelines. The findings of this review endorse the need for targeted strategies to overcome the lack of role clarity of patients in IPC and the power imbalances between patients and HCPs.

\section{BACKGROUND}

Healthcare-associated infections (HCAIs) represent a major risk to patient safety and significantly contribute to increased morbidity, higher mortality rates, prolonged hospitalisations, long-term disability and
Strengths and limitations of this study

- This study used rigorous scoping review methods, including a detailed search of multiple databases (with peer-reviewed literature), grey literature that complied with standards for the conducting and reporting of reviews, and a bidirectional citation chasing approach was used as a supplementary search step.

- Our research adopted an integrative approach to provide an overview of what is known and what the trending topics are in empirical and grey literature about patient involvement in the implementation of infection prevention and control (IPC) guidelines and interventions.

- Identification of gaps in the knowledge about how to operationalise a fundamental change to IPC, from being solely the healthcare professionals (HCPs) responsibility to one that involves a collaborative relationship between HCPs and patients.

- The quality of evidence, that is, part of systematic reviews, was not assessed in this review as in other scoping reviews.

- A lack of standardised language around some key terms could mean some studies were not identified and papers were limited to hospital settings.

increased resistance to antimicrobials, resulting in a substantial financial burden on health services. ${ }^{1}$ HCAIs are the most frequent complication for patients receiving healthcare, with pooled prevalence rates of $7.6 \%$ in high-income countries and $10.1 \%$ in middle to low-income countries. ${ }^{1}$ Despite the high incidence rates, it is estimated that $30 \%-70 \%$ of all HCAIs are preventable. ${ }^{2}$ The failure to adhere consistently to infection prevention and control (IPC) guidelines is a key factor in maintaining the high rates of HCAI occurrence, with healthcare professionals (HCPs) 
average compliance rates with hand hygiene guidelines standing at just $38.7 \% .^{3}$

Many authors stress the need to increase patient involvement in IPC implementation in healthcare settings and when developing new guidelines and initiatives. ${ }^{4-7}$ It is believed that this will ensure a more patient-centred service that prioritises their needs ${ }^{8}$ and increases patient safety by empowering them to take control of their own IPC and increases compliance of HCPs with guidelines. ${ }^{9} 10$

Even when patients are aware of their potential contribution to IPC, their involvement can be undermined by an apprehension about asking or getting involved. ${ }^{11} 12$ Several publications suggest that patients can feel that it is not their responsibility to ask about IPC. They can also perceive that HCPs have enough expertise to recognise the importance of standard procedures in HCAI prevention without having to raise the subject. ${ }^{13} 14$

Current studies on HCAI have provided valuable insights on how to overcome existing barriers to patient involvement. ${ }^{61516}$ However, few of them have mapped the existing strategies to involve patients in the implementation of HCAI guidelines and IPC initiatives across different healthcare settings that go beyond the hand hygiene compliance context. Therefore, the aim of this scoping review was to describe the strategies that have been employed to foster patient involvement in the implementation of IPC guidelines and associated interventions.

\section{METHODS}

\section{Study design}

To identify publications in both peer-reviewed and grey literature, and provide a broad overview of strategies to support patient involvement in the implementation of IPC guidelines, a scoping review was undertaken. The 'scoping' approach helps to generate an overall map of the evidence that has been produced, to clarify working definitions underpinning a research area and/or the conceptual boundaries of a topic. ${ }^{17}$ Therefore, scoping reviews differ from systematic reviews which focus on the effectiveness of a particular intervention based on predetermined outcomes. However, scoping reviews can also be systematic and follow methodological frameworks, such as the one provided by the Joanna Briggs Institute ${ }^{17}$, which is internationally recognised.
The research question that oriented this scoping review was: What are the existing strategies or interventions to support patient involvement in the implementation of IPC guidelines and associated interventions?

\section{Inclusion criteria and types of sources}

The inclusion and exclusion criteria are shown in table 1. Search limit included a 5-year date restriction (2013-2018).

\section{Search strategy and database search}

The search terms were generated based on consideration of the 'participants' (health service users and informal carer for a service user), the 'concept' under investigation (patient involvement in interventions and clinical guidelines) and the 'context' (HCAI and IPC).

\section{Bidirectional citation chasing}

We used a bidirectional citation chasing or pearl growing approach to generate a full list of references pertaining to patient engagement with IPC guideline implementation (figure 1). The pearls in this instance were the two papers sourced in the database search ${ }^{18} 19$ and through the citation chasing process, nine new papers were identified. ${ }^{5-79-11 ~ 20-22}$

\section{Grey literature}

Following a search of the grey literature on Google Scholar using the terms "patient involvement' ${ }^{23}$ or 'guidelines' ${ }^{24}$ or 'HCAI ${ }^{25}, 207$ articles were screened from a total of 21 pages reviewed. However, of the 12 that merited inclusion for data extraction, only three were new papers ${ }^{26-28}$ (figures 1 and 2). The searches for peer-reviewed literature and grey literature were initially undertaken in March and April 2018 and updated in July 2018. A detailed definition of participants, concept and context alongside their respective search terms are described in table 2.

The summary of our search processes, screening and data analysis is available as an online supplementary file. As part of our data analysis, a word cloud was developed to aid the identification of trending topics in the literature (figure 3).

Empirical data from the literature were extracted by $\mathrm{HA}$ and a sample was subsequently cross-checked by JH to ensure consistency. This process was repeated for the

\section{Table 1 Inclusion and exclusion criteria}

\begin{tabular}{ll}
\hline Inclusion criteria & Exclusion criteria \\
\hline $\begin{array}{l}\text { Published in English, Portuguese, } \\
\text { Spanish or French. }\end{array}$ & $\begin{array}{l}\text { Papers were excluded if they reported on HCAl guideline } \\
\text { recommendations, simply cited the importance of service-user } \\
\text { Articles in peer-reviewed journals. }\end{array}$ \\
$\begin{array}{l}\text { Report of evidence focused on: } \\
\text { implement, or reported on broad experiences of HCAl guideline }\end{array}$ \\
$\begin{array}{l}\text { Patient/family involvement patient/family participation in the } \\
\text { implementation of healthcare-associated infection (HCAl) }\end{array}$ \\
$\begin{array}{l}\text { guidelines. } \\
\text { Strategies used to support patient/family involvement in the }\end{array}$ \\
\hline
\end{tabular}




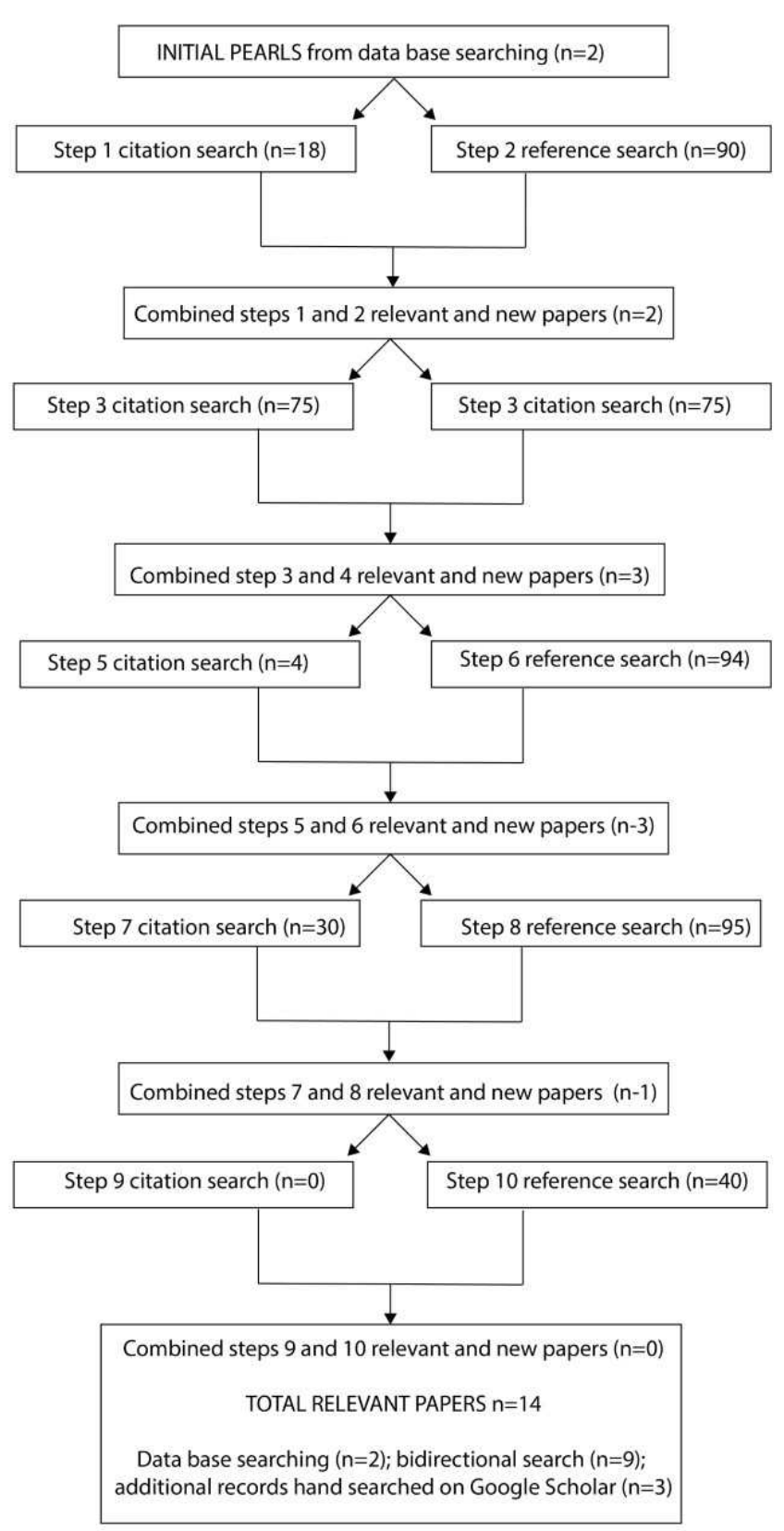

Figure 1 Bidirectional citation searching structure and results.

grey literature. Full details of data extraction can be seen in tables 3 and 4 .

\section{Patient and public involvement}

Patients or the public were not involved in this research.

\section{FINDINGS}

\section{Characteristics of studies}

Country of origin

The country of origin for primary authors was Australia $(\mathrm{n}=6)$, USA $(\mathrm{n}=3)$ and UK $(\mathrm{n}=3)$. The remaining studies were from China and Netherlands (both $\mathrm{n}=1$ ).

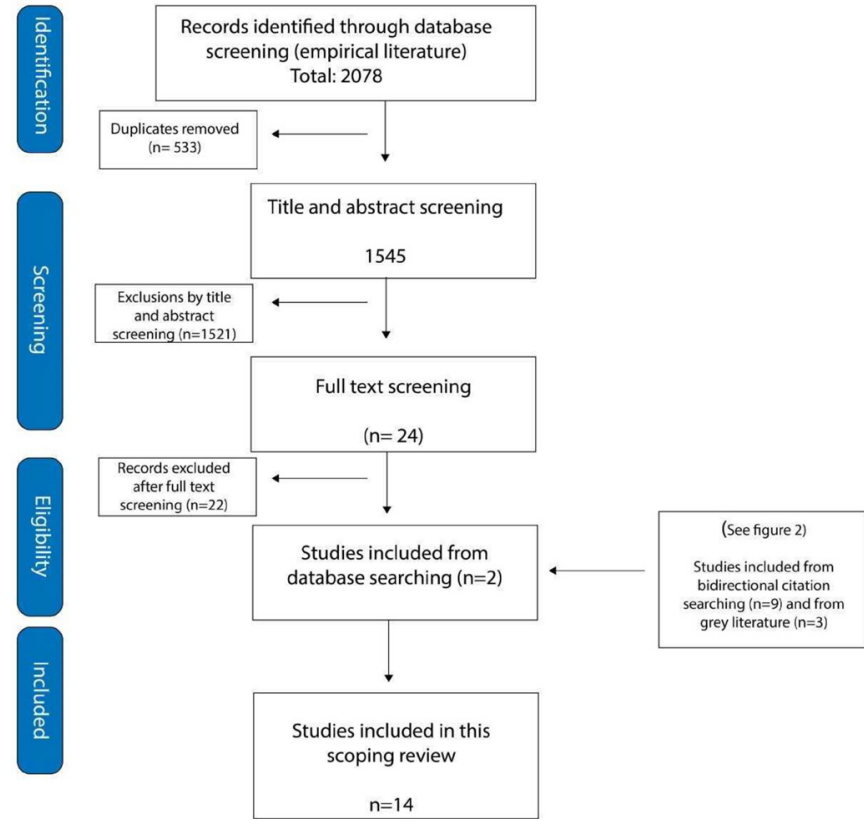

Figure 2 PRISMA flow chart of identification and inclusion of studies. PRISMA, Preferred Reporting Items for Systematic Reviews and Meta-Analyses.

\section{Study participants}

The studies explored both patients' and healthcare providers' roles in preventing and controlling HCAIs.

\section{Type of studies}

Fourteen papers from the international literature search were reviewed (table 3 ), $\operatorname{six}^{5{ }^{6-11}}$ of which were literature reviews (eg, systematic, lexical and integrative), six studies $^{719} 21-2628$ that used qualitative approaches (eg, individual interviews and focus groups), one quasi-experimental study ${ }^{20}$ and an expert panel report. ${ }^{18}$

\section{Trending topics}

Combined word frequencies in all included papers indicate that: patient(s) $2.61 \%$, infection(s) $1.13 \%$, hand(s) $1.14 \%$, hygiene $0.64 \%$, catheter (s) $0.79 \%$, control $0.52 \%$, hospital $0.47 \%$, prevention $0.27 \%$, empowerment $0.21 \%$, involvement $0.21 \%$ were the trending topics in studies of patient involvement in the implementation of IPC guidelines and associated interventions (figure 3 ).

One of the most common words used in the included papers was 'hands'. Appropriate hand hygiene of HCPs is regarded as the single most effective way to protect patients against HCAI and reduce the spread of antimicrobial resistant bacteria. ${ }^{3}$ In our study, all selected papers discussed hand hygiene compliance or had a specific focus on it. ${ }^{69111920}$ However, the implementation of IPC guidelines is not limited to hand hygiene compliance.

\section{Thematic analysis}

The results of thematic analysis revealed three themes pertaining to patient involvement in the implementation of IPC guidelines: (1) Patients' roles in IPC interventions; (2) Levels of patient involvement and (3) Barriers 
Table 2 Definition of participants, concept and context and their respective search terms

\begin{tabular}{|c|c|c|}
\hline Participants & Concept & Context \\
\hline $\begin{array}{l}\text { Patients and family members: Health service } \\
\text { users included patient, family, and those who care } \\
\text { (informal carer) for a service user. }\end{array}$ & $\begin{array}{l}\text { Patient involvement in interventions and clinical } \\
\text { guidelines: } \\
\text { Patient and family involvement refers to 'activity } \\
\text { that is done 'with' or 'by' patients or members of } \\
\text { the public rather than 'to', 'about' or 'for' them' }{ }^{23} \\
\text { Guidelines refer to 'systematically developed } \\
\text { evidence-based statements which assist } \\
\text { providers, recipients and other stakeholders } \\
\text { to make informed decisions about appropriate } \\
\text { health interventions'. }{ }^{4}\end{array}$ & $\begin{array}{l}\text { Healthcare associated infection (HCAI) and } \\
\text { infection prevention and control (IPC): } \\
\text { HCAI refers to 'an infection occurring in a patient } \\
\text { during the process of care in a hospital or other } \\
\text { healthcare facility which was not present or } \\
\text { incubating at the time of admission'. } \\
\text { IPC refers to 'a scientific approach and practical } \\
\text { solution designed to prevent harm caused by } \\
\text { infection to patients and health workers'. }{ }^{25}\end{array}$ \\
\hline $\begin{array}{l}\text { Search terms: } \\
\text { Patient OR client OR 'family member' OR relative }\end{array}$ & $\begin{array}{l}\text { Search terms: (Implement* OR introd* OR uptake } \\
\text { OR utilis* OR utiliz* OR complian* OR concord* } \\
\text { OR adhere* OR disseminat* OR adopt* OR } \\
\text { translat* OR appl* OR 'diffusion of innovation' OR } \\
\text { barrier }^{\star} \text { OR facilitator } \text { Or enabler }^{\star} \text { ) } \\
\text { AND guideline }\end{array}$ & $\begin{array}{l}\text { Search terms: (Infection N3 (healthcare OR } \\
\text { 'health care' OR health care OR hospital OR } \\
\text { nosocomial Or resistant OR antibiotic OR control } \\
\text { OR prevention)) OR (pathogen N3 (healthcare } \\
\text { OR 'health care' OR health care OR hospital OR } \\
\text { nosocomial OR resistant OR antibiotic OR control } \\
\text { OR prevention)) OR 'Alert organism*' OR 'cross } \\
\text { infection' OR cross-infection' OR 'HAl' OR HCAl' } \\
\text { OR 'Methicillin resistant Staphylococcus aureus' } \\
\text { OR 'MRSA' OR 'M.R.S.A.' OR 'Clostridium } \\
\text { difficile' OR 'C. difficile' OR C. difficile' OR 'C. diff'' } \\
\text { OR 'C. diff' OR 'multidrug resistant organisms' } \\
\text { OR 'MDRO' OR 'M.D.R.O.') }\end{array}$ \\
\hline
\end{tabular}

in the professional-patient relationship (figure 4). Patient involvement varied from being real partners to pseudopartners.

\section{Patient role in IPC}

There is a consensus in the studies that both patients and HCPs should jointly advocate for a culture of patient involvement in reducing the burden of HCAIs. However, the extent to which patients should be involved and their role in IPC interventions are not clearly defined. In general, patients can play different roles: potential transmitters of infections, active/passive supporters of IPC, to

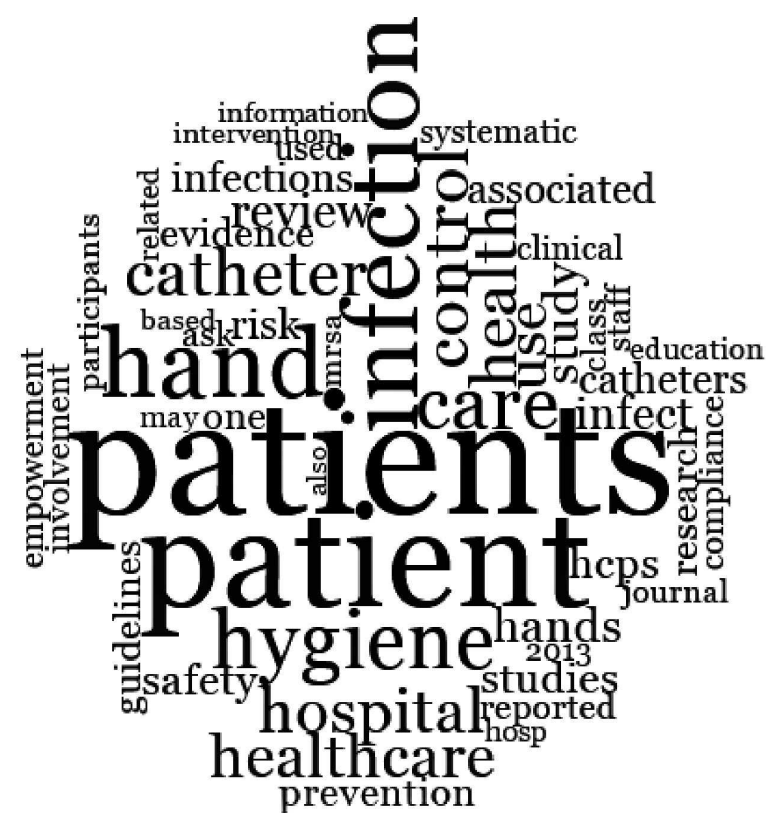

Figure 3 Word cloud ('Wordle') generated in NVivo based on 14 papers selected for scoping review of patient involvement in infection prevention and control guidelines. full partners in IPC. Some tensions emerge from these different roles, such as vulnerability versus responsibility and real partners versus pseudopartners.

\section{Vulnerability versus responsibility}

Concerns have been raised that involving patients in IPC interventions could increase patient anxiety and place responsibility on an already vulnerable person. ${ }^{11}$ Indeed, patients can feel initially shocked, confused and anxious when diagnosed with an infectious micro-organism. They also do not want to feel guilty and responsible for the transmission of infection to others. ${ }^{26}$ Vulnerability versus responsibility in infection transmission is the first tension regarding patient involvement in IPC.

Raising patient's self-awareness on the risks of contamination and cross-transmission of micro-organisms is one of the methods of promoting patient involvement in IPC. ${ }^{27}$ However, our findings suggest that patients are more often acknowledged in their vulnerable role than viewed as potential players in the prevention of infection transmission. Transmission of HCAI through the contamination of patients' hands, for example, is as important as contamination of HCP's hands. ${ }^{11}$ However, the majority of studies have been focused only on strategies to encourage patients to ask HCPs about their compliance with standard precautions, ${ }^{5-7911182021}$ undermining the development of a patient's own accountability for IPC. Only three studies were identified that reported strategies to encourage patients to monitor themselves in IPC 22628 ; for example, with patient-to-patient education. A common characteristic between these three studies was the involvement of long-term care patients. Patients in dialysis clinics, ${ }^{28}$ for example, can be seen as more likely to be engaged in meaningful partnerships on IPC than those admitted for shorter stays. The oral culture of dialysis clinics (eg, with patients talking in the lobby) facilitates the exchange of 


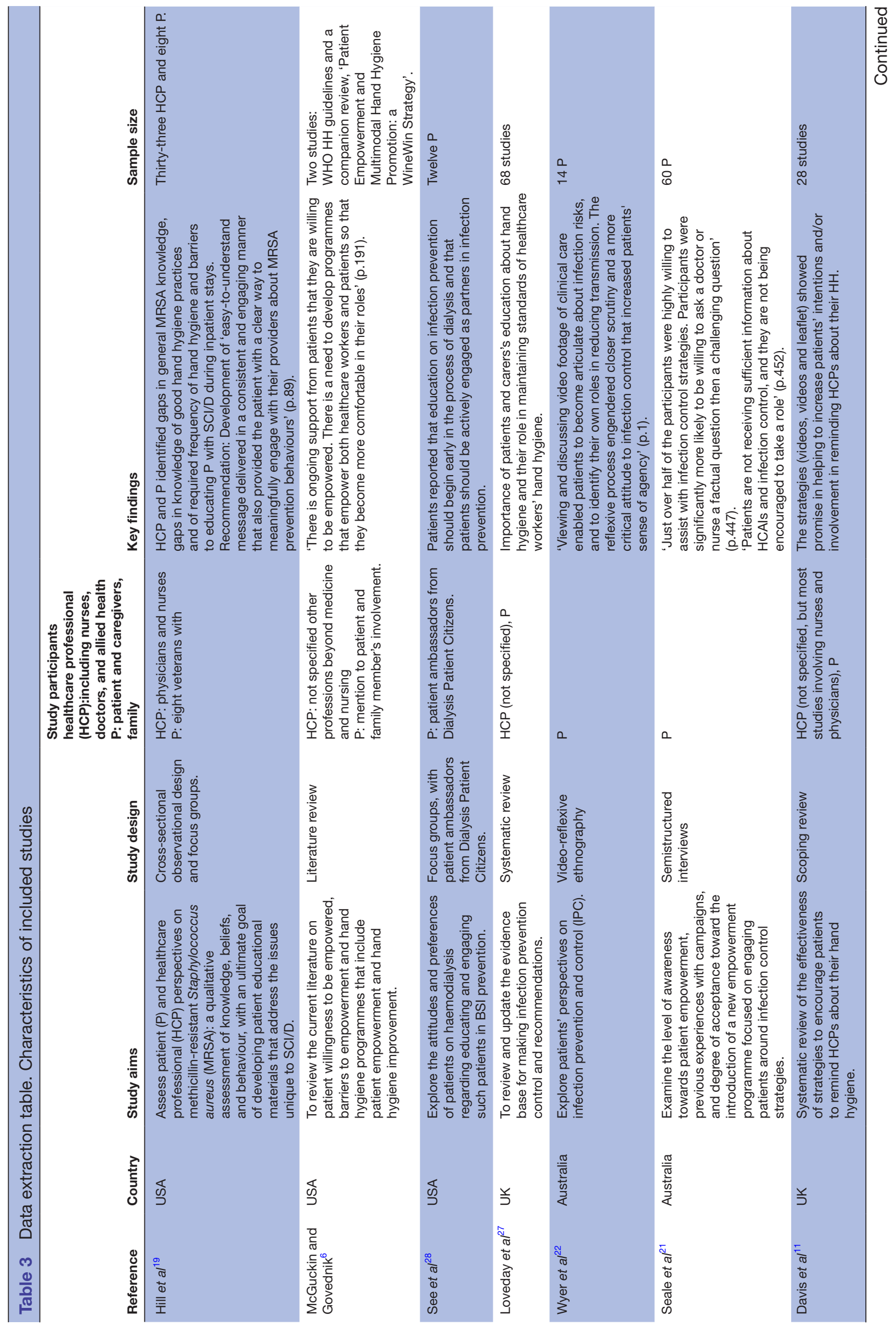




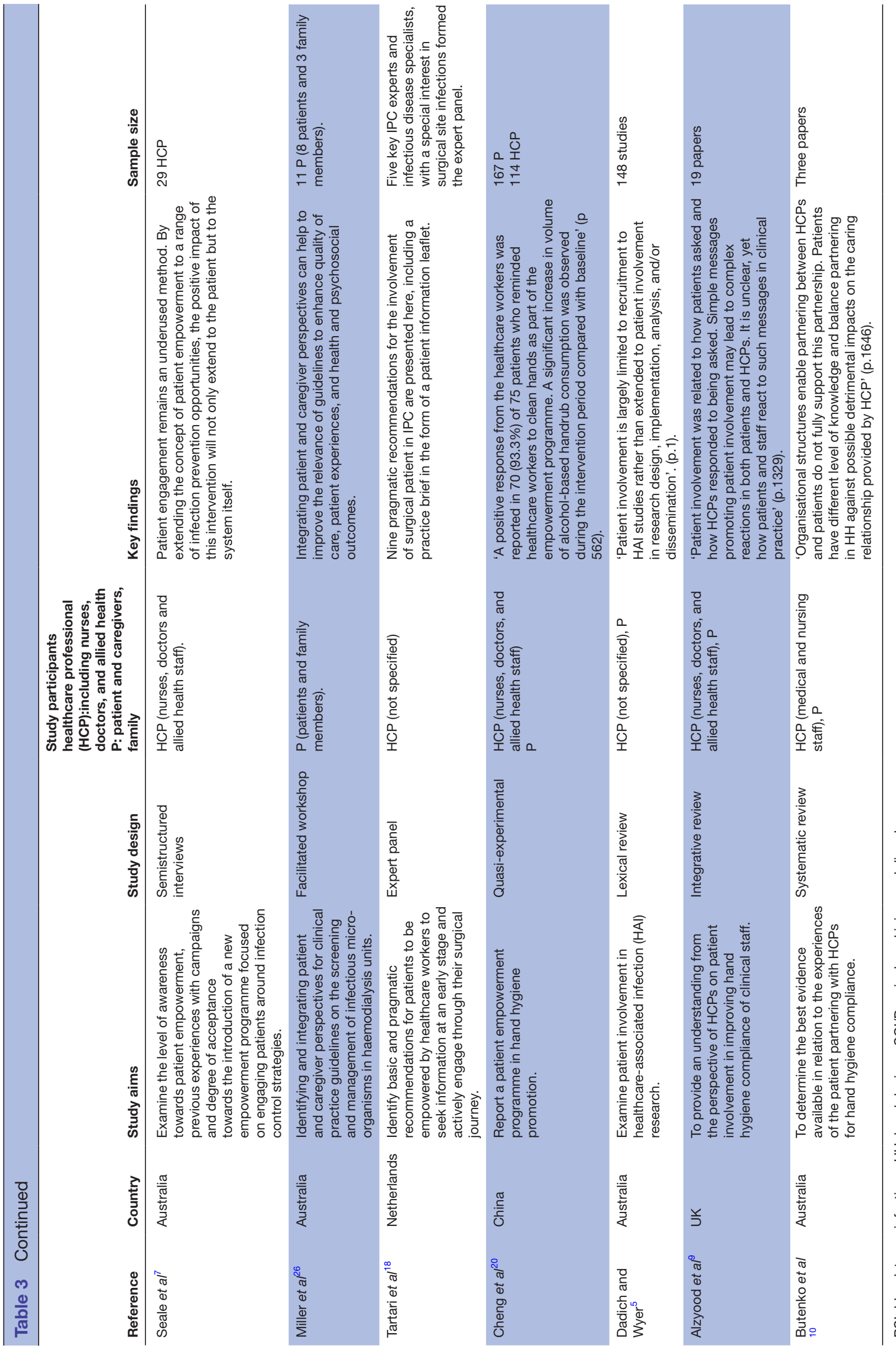




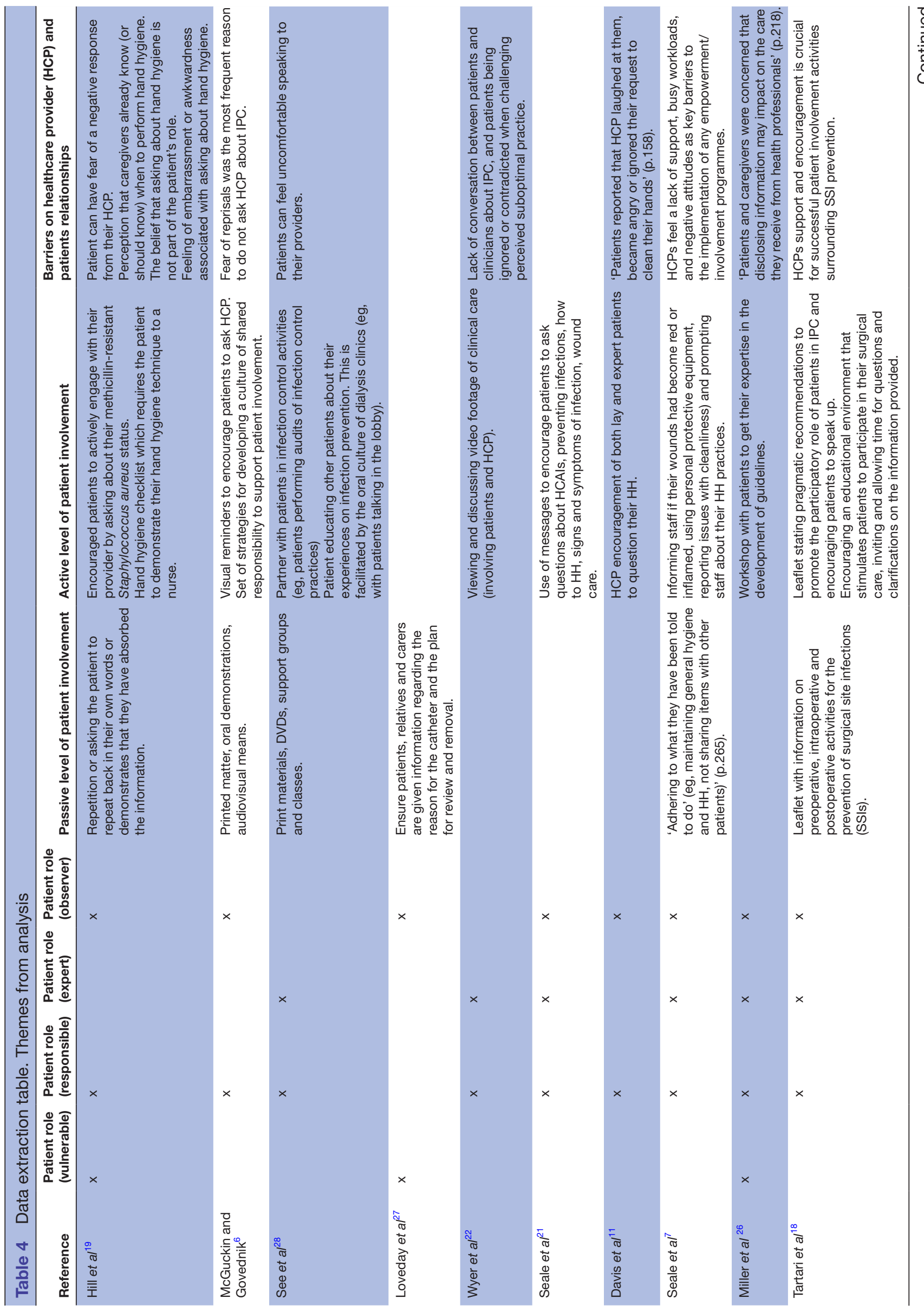


knowledge on IPC between patients and enables them to monitor themselves by sharing stories of their own experiences of the consequences of suboptimal dialysis catheter care. $^{28}$

\section{Real partners versus pseudopartners}

The second tension we identified rests in the dual role of patients in IPC interventions was real partners versus pseudo partners in IPC. This tension could partially be explained by the motivations behind patient involvement. On the one hand, patients are encouraged to get involved in IPC interventions to ensure that their perspectives and knowledge are taken into account to promote safe-care ${ }^{6}$; on the other hand, the rationale for involving patients in IPC interventions is to enable continuous monitoring of HCP practices without the need for additional staff or resources. ${ }^{11}$ On the partnership continuum, patients can be seen both as: (1) coresponsible partners with HCPs for patient safety and part of the solution, through the monitoring of both HCP's behaviours and their own towards IPC or as (2) pseudopartners with an outsider perspective which involves observing what is happening, possibly reporting but not being seen as a true partner in IPC.

In spite of the reported willingness of patients to get involved as real partners in IPC, ${ }^{6} 91021$ our findings revealed that patients can feel more comfortable playing a supportive role (monitoring HCP's behaviours) rather than assisting with infection control strategies. ${ }^{21}$ However, the patient role can be undermined by a patient's assumption that it is not their responsibility to ask about IPC behaviours ${ }^{9}$ and by patients assuming that HCPs know the importance of standard precautions. ${ }^{1011} 19$ Our findings highlight patient reservations, embarrassments and fears associated with asking HCPs about IPC 2671019202226 or impeding their role as partners equally responsible for IPC. When considering partnering for IPC, patients reported different levels of comfort associated with the perceived level of authority and the HCP's role; for example, some patients reported feeling more comfortable asking a nurse about ICP rather than a physician. ${ }^{10}$ Hence, patients may require explicit permission by professionals to share with them the responsibility for IPC. ${ }^{6}$

Although some HCPs report they would be happy for a patient to remind them to wash their hands, they also admit that such conversations could be detrimental to the professional-patient relationship. ${ }^{9}$ Professionals may not support patients asking them about IPC, believing it will create conflict by implying a judgemental perspective and a lack of trust in HCPs to deliver safe care. ${ }^{71}$ Given the diversity of hospital patients and their capacities to be involved, for example, on the basis of severity of illness and cultural background, attempts to involve patients are not always perceived as appropriate. In some cultures, patient reminders can be considered an unacceptable source of confrontation. ${ }^{29}$ Likewise, asking patients to remind HCPs to cleanse hands can be seen as a behaviour contrary to the social norms that occur in healthcare settings. ${ }^{30}$ The relationship between the patient and HCP 


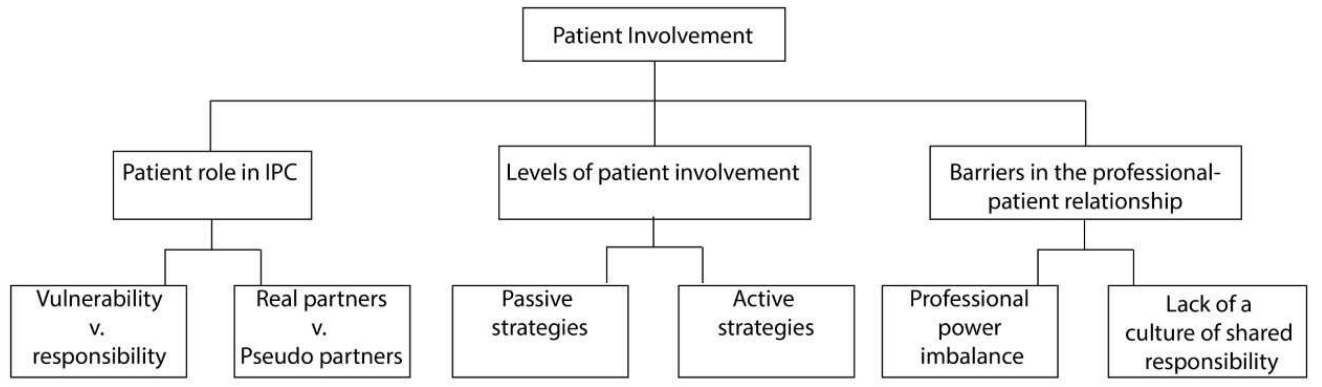

Figure 4 Thematic map highlighting the overarching theme (patient involvement in the implementation of infection prevention and control (IPC) guidelines and associated interventions) and subthemes of analysis.

in the context of IPC reflects existing challenges in the whole organisation of care including power imbalances and clinical dominance that can impact negatively on HCP and patient partnerships. ${ }^{721}$

Building partnerships and collaborative relationships in healthcare requires a core component of role clarity. Our findings suggest a lack of clarity about the role of patients in IPC. This lack of role clarity results in tensions which can impact on the way patient involvement strategies are designed and delivered.

\section{Levels of patient involvement}

To encourage patients to partner with HCPs and be equally responsible for IPC, patient involvement interventions have been developed. Most of these strategies are aimed at empowering patients in IPC. ${ }^{7022} 26$ McGuckin and Govednik $^{6}$ argue that one cannot participate, be involved or be engaged without the components of empowerment including knowledge, skills and an accepting environment. Strategies for involving patients in IPC can vary in terms of topic areas covered and levels of patient participation; these range from relatively passive strategies to active participation in IPC.

Passive strategies, such as written information and audiovisual teaching, were described as potential tools to minimise risk of infection and promote patient engagement with IPC. ${ }^{27}$ In these strategies, patients and relatives were provided with information on IPC recommendations, such as hand hygiene and other standard precautions. ${ }^{5-7} 1118-2031$ Although important, these initiatives are criticised ${ }^{9}$ as they tend to limit patient involvement to adhering to what they are told to do rather than promoting patients as real partners for IPC.

Active strategies promote patient involvement beyond the development of patient's knowledge and skills for IPC taking into account the patient's beliefs and experiences. ${ }^{919}$ Taking patient beliefs into account can help to ensure that patients and HCPs have the same expectations. If patients believe that infection transmission cannot be prevented, they might assume that an active patient role would not help in the prevention of the spread of infection. ${ }^{11}$ When acknowledged in an active role, patients can provide additional insights into the development of IPC guidelines ${ }^{5}$ and become educators themselves. ${ }^{7}$ Some examples of active strategies are video reflexive sessions, ${ }^{22}$ patient-to-patient education, encouraging patients to monitor their own care ${ }^{26}$ and demonstrations followed by discussions on IPC. ${ }^{22}$

Both passive and active strategies require institutional prompts and staff training on how to communicate effectively with patients. ${ }^{679}$ HCP preparedness and institutional support are essential to promote a shift in how patient involvement is understood, that is, from a personal challenge regarding the care provided by an HCP to an organisationally supported mechanism for enhancing patient safety. ${ }^{9}$ This organisational shift can be facilitated by combined strategies of patient empowerment, education and encouragement. ${ }^{18}$ However, the professional-patient relationship and its intrinsic power imbalances remain as the main challenge to real professional-patient partnerships in IPC. ${ }^{92}$

\section{Barriers in the professional-patient relationship}

Our findings revealed that both professionals and patients could feel uncomfortable sharing the responsibility to control and prevent HCAIs. Patients' intentions to better understand and engage in IPC may be negatively misinterpreted by HCPs. The degree of involvement and participation of patients in IPC is linked to both the extent to which they feel comfortable questioning authority and the quality of the relationship.

Two main barriers were described as: (1) relationship power imbalance and (2) lack of an organisational culture of shared responsibility. These are evidenced by a lack of conversation between patients and HCPs about IPC, as well as patients being ignored or contradicted when challenging perceived suboptimal practice. ${ }^{22}$ To overcome such barriers, some initiatives are described in the literature as dispersion of a professional's power and developing a culture of shared responsibility.

To disperse a professional's power, it is recommended that HCPs explicitly invite patients to engage with staff members and to remind them of their IPC duties. ${ }^{828}$ Effective communication between patients and HCPs is highlighted as a key aspect to address power imbalances. Seale et $a l^{7}$ note that communication with patients on issues around IPC should be initiated at the earliest possible opportunity. Training programmes are recommended to 
enable HCPs to communicate with patients and be more responsive to patient concerns without taking offence. ${ }^{32}$ These training programmes and communication strategies can serve the function of addressing the power imbalance and HCP's perception of control over to patients, thus creating a more collaborative partnership. ${ }^{67}$ Second, the literature reports some successful strategies for developing a culture of shared responsibility to support patient involvement.

Multimodal approaches for IPC, comprising patient education and encouragement by HCPs, are part of a culture of shared responsibility in IPC. ${ }^{911} 182021$ These multimodal programmes are included in what McGuckin and Govednik ${ }^{6}$ describe as a key strategy for changing the culture around hand hygiene compliance. Alongside multimodal programmes, the authors describe key steps to patient involvement in the implementation of IPC interventions, these include: a review of the patient's and HCP's willingness to be involved; identification of potential role models to assist in improving the culture of shared responsibility for improving IPC; constant evaluation of barriers and facilitators to patients' and HCP's involvement at the institutional level and; to ensure key decision makers address such barriers.

Butenko et $a l^{10}$ endorse the necessity for changes in cultural beliefs and behaviours to fully support patient involvement in IPC. They state that, although organisational structures to enable partnering between HCPs and patients for hand hygiene compliance exist, the prevailing culture can act as an impediment to the successful implementation of IPC interventions.

\section{DISCUSSION}

Patient empowerment is based on the principles of shared responsibility and the building of partnerships between HCPs and patients. Establishing partnerships and collaborative relationships require a core component of role clarity. Our findings suggest that the role of the patient in IPC remains unclear and the existing efforts to involve them vary from passive to active strategies. Furthermore, these strategies are challenged by culturally engrained barriers in professional-patient relationships, such as power imbalances and clinical dominance.

In optimal real patient involvement, the process of clinical dominance is weakened, and HCPs are encouraged to relinquish their need to control their patients and the spread of HCAI by themselves. Instead, HCPs respect the patient's central role in provision of care and encourage and support them to take responsibility for themselves and others in the context of IPC. One example of this real patient involvement is the use of video for reflexive sessions in which patients are given the opportunity to comment freely on videoed clinical care interactions and feedback their insights to HCP who care for them. ${ }^{33}$

Analysis of the professional-patient relationship shows the professional power issues enunciated in the literature. ${ }^{34}$ Reeves $e t a l^{55}$ noted that, even when developments appear to shift attention towards the patient and their family, there is continuous need to consider the nature of a patients 'role within health and social professions in which 'the balance of power between patients and professionals has traditionally favoured the latter' (p.42). The twinned concept of power/knowledge is often discussed in the literature, advocating the need to increase patient understanding of their own health and care. ${ }^{34}$

Foucault examined the links between knowledge and power and discussed that professionals tend to use their knowledge as a way to control the 'body' of the patient. ${ }^{36}$ Empowering citizens is essential to give them knowledge of their bodies and health conditions and to be able to make decisions in a citizen's action, ${ }^{37}$ which implies patients acting in their role as advocates on their own behalf and being responsible for keeping themselves healthy. Therefore, providing patients with knowledge of IPC, including information about and rationale for standard IPC recommendations, is a means of ensuring their active role, advocacy and responsibility in preventing HCAIs.

However, there is also a need to create an accepting environment for patient involvement in IPC. This would require changes to the predominant organisational culture in which professionals tend to control their organisation's destinies ${ }^{38}$ and also play an authoritarian role over patients. The required cultural changes imply a reversion in the paternalist relation between HCPs and patients, as described by Parsons. ${ }^{39}$ It suggests a need to put patients in a responsible and protagonist role as experts in their own care and IPC, rather than being passive participants and observers of HCPs' behaviours.

\section{CONCLUSION}

This review included 14 papers describing interventions available to support patient involvement in the implementation of IPC guidelines and associated interventions. Our findings endorse the need for patient involvement in IPC and provide insights into a fundamental change to IPC as a common responsibility for both patients and HCPs. This change should be supported by a clear understanding of patient roles, potential levels of patient involvement in IPC and strategies to overcome barriers in the professional-patient relationship (eg, patient encouragement through strategies to promote cultural change and efforts to disperse HCPs' power).

Further studies are needed to understand how to develop and sustain an 'accepting culture' in which patient involvement is not a personal challenge to the care provided by HCPs, but as an essential part of patient safety.

\section{Author affiliations}

${ }^{1}$ Department of Nursing and Midwifery, University College Cork, Cork, Ireland ${ }^{2}$ Department of Clinical Microbiology, Mercy University Hospital, Cork, Ireland ${ }^{3}$ School of Healthcare Sciences, Cardiff University, Cardiff, South Glamorgan, UK ${ }^{4}$ Department of Nursing and Midwifery, Catherine McAuley School of Nursing and Midwifery, University College Cork, Cork, Ireland

${ }^{5}$ Department of Epidemiology and Public Health, University College Cork, Cork, Ireland 
${ }^{6}$ Department of Health, National Patient Safety Office, Dublin, Ireland ${ }^{7}$ Health Service Executive, Cork, UK

Acknowledgements The authors would like to thank the Department of Health and Health Research Board, Ireland for funding this study.

Contributors HA, MM, SC, AB, CNB, DO, DG, ES, FB, JD, MPS, SC, SH, SM, TW and $\mathrm{JH}$ made substantial contributions to conception and design or acquisition of data, or analysis and interpretation of data. $\mathrm{HA}, \mathrm{MM}, \mathrm{SC}, \mathrm{AB}, \mathrm{ES}, \mathrm{SC}, \mathrm{SH}$ and $\mathrm{JH}$ were involved in drafting the manuscript or revising it critically for important intellectual content. HA, MM, SC, AB, CNB, DO, DG, ES, FB, JD, MPS, SC, SH, SM, TW and JH have given final approval of the version to be published. HA, MM, SC, AB, CNB, DO, DG, ES, FB, JD, MPS, SC, SH, SM, TW and JH have agreed to be accountable for all aspects of the work in ensuring that questions related to the accuracy or integrity of any part of the work are appropriately investigated and resolved.

Funding Funding for this study was supported by the Department of Health and the Health Research Board. Applied Partnership Awards, APA-2017-002.

Competing interests None declared.

Patient consent for publication Not required.

Provenance and peer review Not commissioned; externally peer reviewed.

Data sharing statement Requests for further information can be made to the corresponding author on reasonable request.

Open access This is an open access article distributed in accordance with the Creative Commons Attribution Non Commercial (CC BY-NC 4.0) license, which permits others to distribute, remix, adapt, build upon this work non-commercially, and license their derivative works on different terms, provided the original work is properly cited, appropriate credit is given, any changes made indicated, and the use is non-commercial. See: http://creativecommons.org/licenses/by-nc/4.0/.

\section{REFERENCES}

1. World Health Organization. Report on the burden of endemic health care-associated infection worldwide: World Health Organization, 2011.

2. Umscheid CA, Mitchell MD, Doshi JA, et al. Estimating the proportion of healthcare-associated infections that are reasonably preventable and the related mortality and costs. Infect Control Hosp Epidemiol 2011;32:101-14.

3. World Health Organization. WHO guidelines on hand hygiene in health care: World Health Organization, 2009.

4. Allegranzi B, Conway L, Larson E, et al. Status of the implementation of the World Health Organization multimodal hand hygiene strategy in United States of America health care facilities. Am J Infect Control 2014;42:224-30.

5. Dadich A, Wyer M. Patient involvement in healthcare-associated infection research: a lexical review. Infect Control Hosp Epidemiol 2018;39:710-7.

6. McGuckin M, Govednik J. Patient empowerment and hand hygiene, 1997-2012. J Hosp Infect 2013;84:191-9.

7. Seale $H$, Chughtai AA, Kaur R, et al. Empowering patients in the hospital as a new approach to reducing the burden of health careassociated infections: The attitudes of hospital health care workers. Am J Infect Control 2016;44:263-8.

8. Nilsen ES, Myrhaug HT, Johansen M, et al. Methods of consumer involvement in developing healthcare policy and research, clinica practice guidelines and patient information material. Cochrane Database Syst Rev 2006;3:CD004563.

9. Alzyood M, Jackson D, Brooke J, et al. An integrative review exploring the perceptions of patients and healthcare professionals towards patient involvement in promoting hand hygiene compliance in the hospital setting. J Clin Nurs 2018;27:1329-45.

10. Butenko S, Lockwood C, McArthur A. Patient experiences of partnering with healthcare professionals for hand hygiene compliance: a systematic review. JBI Database System Rev Implement Rep 2017;15:1645-70.

11. Davis R, Parand A, Pinto A, et al. Systematic review of the effectiveness of strategies to encourage patients to remind healthcare professionals about their hand hygiene. J Hosp Infect 2015;89:141-62.

12. Ottum A, Sethi AK, Jacobs EA, et al. Do patients feel comfortable asking healthcare workers to wash their hands? Infect Control Hosp Epidemiol 2012;33:1282-4.

13. Michaelsen K, Sanders JL, Zimmer SM, et al. Overcoming patient barriers to discussing physician hand hygiene: do patients prefer electronic reminders to other methods? Infect Control Hosp Epidemiol 2013;34:929-34.

14. Pittet D, Panesar SS, Wilson K, et al. Involving the patient to ask about hospital hand hygiene: a national patient safety agency feasibility study. J Hosp Infect 2011;77:299-303.

15. McGuckin M, Storr J, Longtin Y, et al. Patient empowerment and multimodal hand hygiene promotion: a win-win strategy. Am J Med Qual 2011;26:10-17.

16. Davis RE, Sevdalis N, Pinto A, et al. Patients' attitudes towards patient involvement in safety interventions: results of two exploratory studies. Health Expect 2013;16:e164-76.

17. Peters $M$, Godfrey $C$, Mclnerney $P$, et al. The Joanna Briggs Institute Reviewers' manual 2015: methodology For JBI Scoping Reviews. 2015.

18. Tartari E, Weterings V, Gastmeier P, et al. Patient engagement with surgical site infection prevention: an expert panel perspective. Antimicrob Resist Infect Control 2017;6:45.

19. Hill JN, Evans CT, Cameron KA, et al. Patient and provider perspectives on methicillin-resistant Staphylococcus aureus: a qualitative assessment of knowledge, beliefs, and behavior. J Spinal Cord Med 2013;36:82-90.

20. Cheng VCC, Wong SC, Wong IWY, et al. The challenge of patient empowerment in hand hygiene promotion in health care facilities in Hong Kong. Am J Infect Control 2017;45:562-5.

21. Seale H, Chughtai AA, Kaur R, et al. Ask, speak up, and be proactive: empowering patient infection control to prevent health care-acquired infections. Am J Infect Control 2015;43:447-53.

22. Wyer $M$, Jackson $D$, ledema $R$, et al. Involving patients in understanding hospital infection control using visual methods. J Clin Nurs 2015;24:1718-29.

23. Involve N. Briefing notes for researchers: involving the public in NHS, public health and social care research. UK: INVOLVE Eastleigh, 2012.

24. World Health Organization. Guidelines for WHO guidelines. World Health Organisation. Geneva, Switzerland: World Health Organisation, 2003.

25. World Health Organization. Clean care is safer care. $2018 \mathrm{http} / / /$ www.who.int/gpsc/ipc/en/

26. Miller HM, Tong A, Tunnicliffe DJ, et al. Identifying and integrating patient and caregiver perspectives for clinical practice guidelines on the screening and management of infectious microorganisms in hemodialysis units. Hemodial Int 2017;21:213-23.

27. Loveday HP, Wilson JA, Pratt RJ, et al. epic3: national evidencebased guidelines for preventing healthcare-associated infections in NHS hospitals in England. J Hosp Infect 2014;86:S1-70.

28. See I, Shugart A, Lamb C, et al. Infection control and bloodstream infection prevention: the perspective of patients receiving hemodialysis. Nephrol Nurs J 2014;41:37.

29. Ho ML, Seto WH, Wong LC, et al. Effectiveness of multifaceted hand hygiene interventions in long-term care facilities in Hong Kong: a cluster-randomized controlled trial. Infect Control Hosp Epidemiol 2012;33:761-7.

30. Stewardson AJ, Sax H, Gayet-Ageron A, et al. Enhanced performance feedback and patient participation to improve hand hygiene compliance of health-care workers in the setting of established multimodal promotion: a single-centre, cluster randomised controlled trial. Lancet Infect Dis 2016;16:1345-55.

31. Seale H, Novytska Y, Gallard J, et al. Examining hospital patients knowledge and attitudes toward hospital-acquired infections and their participation in infection control. Infect Control Hosp Epidemiol 2015;36:461-3.

32. Schwappach DL, Frank O, Davis RE. A vignette study to examine health care professionals' attitudes towards patient involvement in error prevention. J Eval Clin Pract 2013;19:840-8.

33. Wyer M, ledema R, Hor SY, et al. Patient involvement can affect clinicians' perspectives and practices of infection prevention and control: A "post-qualitative" study using video-reflexive ethnography. International Journal of Qualitative Methods 2017;16:1609406917690171.

34. Nicholson Thomas E, Edwards L, McArdle P. Knowledge is Power. A quality improvement project to increase patient understanding of their hospital stay. BMJ Qual Improv Rep 2017;6:u207103. w3042.

35. Reeves S, Lewin S, Espin S, et al. Interprofessional teamwork for health and social care: John Wiley and Sons, 2011.

36. Foucault M. The birth of the clinic: an archaeology of medical perception. Translated by A.M. Sheridan Smith. New York: Vintage Books, 1973.

37. Fox A. Intensive diabetes management: negotiating evidence-based practice. Can J Diet Pract Res 2010;71:62-8.

38. Brannigan ET, Murray E, Holmes A. Where does infection control fit into a hospital management structure? J Hosp Infect 2009;73:392-6.

39. Parsons T. The social system: Routledge, 2013. 